\title{
Revista general de la endometriosis
}

\author{
Doctor Camilo Arango Jaramillo
}

Indudablemente es la endometriosis una de las entidades que en la actualidad ha adquirido mayor importancia, no sólo para el médico en general, sino particularmente para el ginecólogo. Desde los clásicos trabajos de Sapson, hace 33 años, ha sido motivo de intensivo estudio, sólo excedido por el merecido al cáncer cervical. Consecuentemente, el mayor conocimiento de la enfermedad ha traido un mayor número de diagnósticos, considerándose, según palabras de Gardner, "sólo excedida, en mi propia práctica, por los miomas uterinos y las lesiones obstétricas, como indicación para la cirugia mayor", agregandio posteriormente: "En otras palabras, para nosotros, la endometriosis pélvica es mucho más frecuente que los embarazos extrauterinos la tuberculosis genital, el cáncer de cuello y cuerpo uterinos, tumores ováricos y secuelas de enfermedades inflamatorias pélvicas".

Ya desde su primera descripción, Sapsom deirinió la endometriosis como: "la presencia de tejido ectópico, con la estructura histológica y la función de la mucosa uterina, in cual incluye condiciones anormales que pueden resultar no solamente de la invasión del órgano y demás estructuras por el tejido ectópico, sino también por sus relaciones con la menstruación".

En la acaualidad, permaneciendo intactas las líneas generales de la concepción de Sampson, se amplia su localización dentro de la patología, considerándose como una, y la más importante, de las llamadas "enfermedades invasoras de tipo uro-genital", entendiéndose con tal denominación los padecimientos ocasionados por la invasión anormal de los tejidos pelvianos por elementos celulares derivados de los órganos genitales: endometrio, endocervix, endosalpinx, epitelio germinal del ovario y el mismo peritoneo, formando como diferentes capítulos: la endometriosis propiamente dicha, endocervicosis, endosalpingiosis y esclerosis peritoneal. 
Es importante puntualizar que al desarrollarse ias células ectópicas, tienen consigo sus propiedades especificas, o sea su actividad secretora y cíclica, causando no solamente s'nómenos de dislocación funcional, sino también las manifestaciones de los quistes de retención, ya sea de secreciones especificas o de simples liquidos sanguinolentos.

Historia.-Al recordar rápidamente la historia de la endometriosis, vemos a Waideyer en 1870, quien por primera vez encontró invadido el estroma ovárico por el epitelio super jucial. Pero la primera descripción de la enfermedad es de Russel, en 1888, al relatar un caso de "endometroide" del ovario, atribuvendo la presencia del tejido endometrial a inclusiones Mullerianas, tesis apoyada posteriormente por los trabajos de Janney y Blcir.

Martin, en 1891, describió un "adenoma" del iigamento redondo, explicándolo como inclusión de restos Wolfianos, tesis apoyada posteriormente por Von Reklinghausen y ampliada por los trabajos de Cutberth 'y Lockier, y que hoy en dia, como veremos posteriormente, se encuentra rebatida. Cullen, uno de los más grandes investigadores del tema, describió a fines dul siglo pasado el primer caso de adenomioma, reportando posteriormente en 1908 noventa casos de diferentes tipos de endometriosis. Durante el mismo año Pfanestiel reportó un caso de localizacion en el septum recto-vaginal, seguido por dos casos de los iigamentos de Mackenrodt, descritos por Ivanoff, quien plantea su teoria de la metaplasia celómica. En 1921 Sampson sentó las bases firmes para la individualización de la enfermedad y, a raíz de tales trabajos se desencadena una verdadera avaiancha de investigadores, entre los cuales encontramos a Meigs, Halban, Sturgis, Novak, Te Linde, Kelso, Scott, Gardner, Counseller y muchos otros.

Etiopatogenia.-Aunque en la época actual encontramos muchas cpiniones unánimes respecto a diferentes aspectos de la endometriosis, tales como su sintomato'ygía, apariencia macroscó pica, requisitos microscópicos para su iagnóstico definitivo, etc., son muchas las teorias expuestas para explicar la etiología y patogenia de las distintas lesiones, hecho significativo de que ninguna teoria, por sísola, puede aclarar todos los casos y localiza ciones de la enfermedad.

En lineas generales estas teorias pueden dividirse en tres grupos:

1. Las que sostienen que el tejido ectópico es transportado desde el útero hasta su nueva locaización; dentro de este grupo 
están comprendidas las teorias de implantación, transplante y metaplasias.

2. Las que sostienen el desarrollo "in situ" del tejido ectópico a costa de los tejidos locales; dentro de este grupo encontramos jas teorias de la metaplasia celómica, de la metaplasia ovárica, de los restos celulares embriológicos, de ia diferenciación tisular y de la extensión directa.

3. Combinación de las teorías anteriores.

Veamos un ligero recuerdo de las principales.

El primer paso importante para despejar tan intrincado panorama, fueron los trabajos de Cullen para rebatir a Von Reklinghausen, quien sostenía el origen wolfiano de las inclusiones, afirmando que los tumores con tejido glandular se encontraban, con mayor frecuencia en la pared posterior del útero, cerca de la porción ístmica de los tubos de Falopio, sitio en el cuá se encuentran los restos wolfianos con mayor frecuencia. Cullen probó, y demostró, la continuidad directa entre el endometrio y el tejido similar encontrado en la musculatura, en la mayoría de los casos, lo cual hoy se acepta plenamente, según los datos proporcionados por los mejores estudios radioiógicos. Pero lo que es más diciente, casi siempre, aunque sea imposible probar la continuidad, o se trate de focos alejados, tales tejidos ectúpicos reaccionan a la administración de hormonas ováricas, y sus reacciones durante el ciclo menstrual y aun durante el embarazo, son similares a las del endometrio tópico, lo cual no deja duda sobre su primitivo origen mulleriano.

Entre las teorías que sustenta el desarrollo del tejido ectópico a partir de cambios en los tejidos locales, encontramos la teoría celómica o sero-epitelial, expuesta por Ivanoff y sostenida por Meyer, la cual se basa en el mismo origen celomático del epitelio peritoneal y del endometrio; el tejido aberrante se originaria por una diferenciación anormal del epitelio celomático, bajo la influencia de diferentes estimulos que, para algunos, serían los estados irritativos e inflamatorios, lo cual, sin embargs, no concordaría con ciertos argumentos, como la poca frecuencia de la endometriosis en la raza negra, la cual presenta, por el contrario, un altísimo porcentaje de procesos inflamatorios además las secuelas propias de estos estados inflamatorios, iales como adherencias, etc., sólo se presentan tardíamente en la endometriosis, lo cual las calificaria no como causa, sino como una consecuencia de la enfermedad. 
Para otros autores, Meigs entre ellos, el factos: estimulante del cambio y crecimiento del epitelio celómico o de jos restos embrionarios, sería un gran número de ciclos menstruales ininterrumpidos, ya sea por retardo matrimonial, demora en la concepción o uso de medios anticoncepcionales. Jaennert encuentra, además, la frecuente coexistencia de otros factores, tales como la hiperplasia endometrial, poliposis y aun carcinomatosis.

A pesar de la gran cantidad de casos susceptibles de explicación por la teoría celómica, determinadas localizaciones, tales como las vulvares, perineales, etc., no serian aclaradas por ella. Las localizaciones vulvares, vaginales y perineales serian explicadas por una ampliación de la teoria anterior, tal como tratan de hacerlo Williams y Young con su teoría mesencilimatosa, recurriendo a tales tejidos, origen común del epitelio germinativo, celomático y mulleriano, como los puntos de partida para las posteriores transformaciones. Aunque esta teoría busca múltiples posibilidades de cambios celulares y tisulares, sobre la base de células muy diferenciadas, no alcanza a explicar localizaciones tales como las de los brazos y pulmón.

Sampson expone su teoria en la siguiente forma: "La endometriosis ovárica y otras formas de endometriosis peritoneal, provienen de la "implantación" de trozos de mucosa riulleriana, ya sea por origen uterino o tubárico, que han sido arrastrados por la sangre menstrual, escapando por la luz tubaria hacia la cavidad peritoneal, alojándose en la superficie de varias estructuras pélvicas. La mucosa ectópica de estos implantes, aparte de su tamaño y situación, puede volverse un foco adicional para la propagación de la endometriosis, ya sea por propagación directa, o por trozos de tejido mulleriano que se desprencien de ella durante la reacción menstrual". Sampson creia que el ovario era una incubadora para estos implantes, atribuyéndole una gran virulencia, ya que notaba mayores areas de invasión cuando encontraba quistes endometriales en ei ovario, pero hoy en día sabemos que pueden haber lesiones extensivas sin la presencia de endometriosis ovárica. El autor intervino quirúrgicamente, muchos pacientes durante la época menstrual, observando con alguna frecuencia el reflujo de la sangre por el orificio tubario, flujo retrógrado que estaría facilitado por cualquier lesión estenósica cervical, o por las retrodesviaciones literinas. Esta teoría, explicación sencilla de la endometriosis pélvica. se encuentra confirmada por varios hechas: 
1. Comprobación de que el orificio peritoneal de ia trompa se encuentra, con gran frecuencia, permeable.

2. Localización del injerto primario en los sibios declives y vecindades del orificio peritoneal tubárico.

3. Coexistencia muy frecuente de condiciones que impiden la eliminación normal de producto menstrual.

4. Presencia comprobada de sangre y de mucosa endometrial en el interior de la trompa.

5. Existencia de la endometriosis sólo durante el periodo genital de las menstruaciones.

Al lado de estas comprobaciones se han formulado objeciones a la teoría de Sampson, las cuales fueron reccgidas por Novak en la siguiente forma:

1. La menstruación retrógada, cuando puede ocurrir, es un fenómeno de rara observación, contrastando con la gran frécuencia de la enfermedad.

2. Es difícil creer que el endometrio, al desprenderse del útero penetre en el delgado orificio de la trompa, atraviese su pequeñisima luz en contra del peristaltismo normal, siendo aún capaz de implantarse en las estructuras pélvicas

3. El endometrio menstual es, por lo general, degenerado o muerto, por lo cual es difícil creer en su desarollo en sitio tan poco propicio como el peritoneo y los experimentos referentes al trasplante del endometrio, con crecimiento posterior, se refieren a tejidos normales y saludables.

4. Los experimentos de Heyn, creando artificialmente fístulas útero-abdominales, que permitan la llegada libre ce la sangre menstrual a la cavidad peritoneal, han fallado en demostrar el desarrollo ulterior del endometrio.

Pero trabajos posteriores de Te Linde y scott, empleando en sus experimentos técnicas más depuradas, desvirtúan los hallazgos iniciales de Heyn, pues lograron demostrar la viabilidad de los transplantes autónomos y, lo que es más importante. la viabilidad fisiológica del endometrio desprendido.

Una explicación intermedia y muy documentada sería la de Hubner y Phillipp, quienes demostraron en un 85\% de pacientes con endometriosis, la presencia de mucosa uterina en la porción intersticial de la trompa. Según los autores, éstos constituirian el foco primitivo, del cual se desprenderían trozos de mucosa en épocas diferentes de las del periodo menstrual, teniendo, por lo 
tanto, mayores posibilidades de viabilidad. A esta teoria se adhieren Ahumada y Di Paola.

Halban para explicar determinadas localizaciones no aclaradas por otras teorias, sugiere la posibilidad de diseminación por vía vascular o linfática, considerándola más probable aún desde la demostración del poder histolítico del estromacitógeno y su penetración poliposa dentro de los espacios linfáticos. Jaennert, en sus trabajos sobre la patogenesis de la endometricsis, sostiene que las células endometriales benignas normales son susceptibles de diseminación y metastasis por las mismas vías seguidas por el adenocarcinoma endometrial, reportando cinco casos en los cuales fue encontrado tejido endometrial en los nódulos línfáticos removidos durante la intervención quirúrgica. Trata de conciliar varias teorias, sosteniendo que este endometrio benig:lo puede aparecer diseminado y metastásico de varias maneras: extensión directa, exfoliación de células viables, implantación de estas células, metástasis linfáticas a los órganos cercanọs, o hematógenas a los órganos distantes. La propagación linfática parece, sin embargo, una de las menos acaecidas, dada la minima incidencia de endometriosis en el ligamento ancho, por el cual discurre el principal pediculo linfático genital.

Al lado de las múltiples teorias que tratan de explicar la etiología y patogenia de la endometriosis, los diversos autores tampoco han logrado acuerdo sobre cuáles son los factores determi. nantes en estimular el desarrollo de la entidad, pero es lo más lógico pensar que en cada caso particular existe un estímulo propio.

Pero sea cual sea, en un determinado caso, el 1actor que favorece la metaplasia o el desarrollo de los restos embrionarios por mucho tiempo latentes, o la proliferación del tejico recién implantado, o transportado por invasión directa o embolia, existen factores comunes y hechos concretos, como:

1. La existencia del ovario es indispensable para la iniciación de la endometriosis y asímismo, es indispensable $\epsilon$ ? ovario para que continúe la actividad endometriósica.

2. Sea que el ovario actúe por sí mismo o como intermediario entre la prehipófisis y el endometrio, su presencia es esencial, ya que la supresión de la función ovárica, por ablacion quirúrgica; radioactiva o menopausia, determina la rápida regresión de los procesos endometriósicos. 
3. Es indudable que el ovario actúa a través de sus hormonas, siendo de pensar, por la observación clínica y de laboratorio, que serian los estrógenos los fundamentales en la génesis y sostenimiento de la endometriosis, por los caracteres proliferativos de dicha hormona, actuando, ya sea por exceso, como io sugiere la frecuente asociación con la hiperplasia glándulo-quística, o por deficiencia relativa o absoluta de la progesterona.

\section{Clasificación y localizaciones}

Es unánime la opinión de los autores en dividir la endometriosis en interna y externa, estando aquélla constituída por las lesiones en continuidad con el endometrio y ésta, cuando tal continuidad existe, aunque una misma lesión puede participar al mismo tiempo de las caracteristicas de interna y externa. Sin embargo, de común acuerdo, con el término de endometriosis se quiere determinar la variedad externa, reservando el nombre de adenomiosis para la interna.

En las localizaciones internas se describen cuatro variedades, según sus diferentes modalidades de expansión:

1. Adenomiosis difusa, envolviendo toda la matriz y ocasionando aumento simétrico de ella.

2. Areas de adenomiosis localizada, pero sin cápsula, histológicamente idénticas a la anterior, pero localizadas en su crecimiento.

3. Crecimiento submucoso, proyectado hacia la cavidad uterina.

4. Adenomiosis circunscritas, como un mioma cualquiera, encapsuladas, constituyendo un verdadero adenomiona.

Para las localizaciones externas Hoffman recientemente ha formulado una clasificación práctica, basada en la extensión de las lesiones, y especialmente útil desde el punto de vista conducta; en ella se considera que la endometriosis, como los neoplasmas, puede ser pequeña y superficial, o crecer, infiltrándose en profundidad, llegando hasta obstruir vísceras huecas y producir tumefacciones susceptibles de ser confundidas con crecimientos cancerosos. Considera cuatro etapas, asi:

1. a) Limitada a los ligamentos útero-sacros $\mathrm{y} / \mathrm{o}$;

b) Limitada a un ovario $\mathrm{y} / \mathrm{o}$;

c) Implantación peritoneal superficial:

2. a) Invasión extensiva de un ovario con invasion menor del otro $\mathrm{y} / \mathrm{O}$; 
b) Implantaciones superficiales sobre los dos cvarios $\mathrm{y} / \mathrm{o}$;

c) Implantación superficial del Douglas;

d) Lesiones infiltrativas de la superficie uterina o de'los úterosacros.

3. a) Invasión extensiva de los dos ovarios;

b) Quistes ováricos bilaterales;

c) Invasión recto-vaginal profunda;

d) Infiltración intestinal no obstructiva.

4. a) Invasión vesical;

b) Invasión intestinal obstructiva;

c) Invasión ureteral.

En cuanto a la localización de las lesiones, las numerosisimas estadísticas traen la mayor frecuencia en el siguiente orden: ovario, ligamentos útero-sacros, fondo de saco de Durglas y útero, siendo menos numerosas las invasiones al recto, signoides, vejiga y ureteres, y más raras aún en la vagina, periné, nmbligo, cicatrices abdomino-perineales, etc. Recientemente Bungeles y Fleury Silveira, en el Brasil, recogieron unos pocos casos de lesiones endometriales en la cavidad pleural, y Navratil y Kreamer pudieron comprobarla en el antebrazo, sobre los músculos extensores del carpo.

\section{Patología}

El aspecto macroscópico de las lesiones, tal como se observan en el curso de las laparotomias, puede presentar todas las gamas: desde simples cambios de coloración peritoneal, hasta los grandes quistes de "mora" o "chocolate", con todo su séquilio adherencial e irritativo. Habrá depósitos de sangre antigua en el fondo de saco de Douglas, en los casos de perforaciones por gran presión intraquística, y cantidades muy discretas de sangre no coagulada en los casos que no han sufrido la perforación, contingencia relativamente rara, por las organizaciones y retracciones del material intraquistico. En los casos de continuos derrames, el peritoneo adquiere aspecto grueso e hiperhémico.

Los cuadros histológicos no siempre pueden apreciarse con claridad, a consecuencia de las deformaciones tisuiares sufridas por los factores de presión, irritación, etc. Estas figuras tienen. gran variedad: el desarrollo ectópico puede estar formado sólo por estroma, constituyendo la endometriosis intersticial, o por estroma y tejido glandular, formando los casos mixtos, los cuales casi siempre se encuentran en las formaciones ováricas. El estroma 
rodea al tejido glandular, parcial o totalmente, apreciándose $\mathrm{e}$ : epitelio cilíndrico y bajo, o cilíndrico y alto, respectivamente, siendo esta dependencia de la altura epitelial una característica típica de la lesión, según la mayoria de los tratadistas.

Cuando el endometrio crece hacia la profundidad, en zonas donde hay tejido muscular, estimula el crecimiento de la fibra lisa y simula, histológicamente, la típica figura de una adenomiosis.

Con gran frecuencia se observan cambios hormonales, y aun deciduales, aunque es más común la morfología puramente proliferativa del endometrio ectópico, mostrando sólo excepcionalmente cambios progestacionales.

En aigunas acasiones el epitelio de los quistes recuerda el epitelio tubárico con la presencia de células ciliadas $y$ no ciliadas Ya Sampson y Everett las habian descrito, y usaron por primera vez el término endosalpingiosis. Posteriormente otros investigadores, entre ellos Te Linde, han encontrado zonas en las cuales se puede apreciar el típico epitelio tubárico, alternadas o no, con típico epitelio uterino. También se han encontrado, auncuales se puede apreciar el típico epitelio tubárico, alternadas o no, con típico epitelio uterino. También se han encontrado, aunque más raramente, glándulas cervicales penetrando dentro del músculo uterino y proliferaciones fuera de él, lo cual constituiría una prueba de la real existencia de la endocervicosis. Alvarez Bravo describe estas lesiones, lo mismo que las formaciones nodulares de endometrio, en especial el casi patognomónico nóduio del tabique rectovaginal.

En los últimos tiempos se describen cada vez con mayor frecuencia lesiones de esclerosis peritoneal, independientes 0 asociadas con lesiones endometriósicas, situadas en forma preferente sobre el peritoneo pélvico infraumbilical, especificidad topográfica que sería una manifestación de hiperestrinismo, pues como es bien sabido, sólo el peritoneo infraumbilical reacciona a la influencia de los estrógenos.

Aunque muy raramente pueden encontrarse focos endometriósicos asociados con lesiones malignas, especialmente con aquellas del tipo adenocarcinoma.

Como ya lo anotamos, el reconocimiento histológico de la lesión es, en muchas ocasiones, muy difícil, ya sea por las causas expuestas, o según la opinión de Novak y De Lima: "el endometrio aberrante de la endometriosis o adenomiosis es por lo gene- 
ral de una variedad inmadura, lo cual se pone en evidencia por el hecho de sólo responder a las infiuencias estimulantes de crecimientos, determinadas por ios estrógenos, y no a la influencia diferenciadora de la progesterona”.

\section{Clínica}

La sintomatologia de la endometriosis puede piesentar todo un espectro, desde los casos totalmente silenciosos, hasta aquellos correspondientes a las gruesas lesiones que han ocasionado obstrucciones o estenosis orgánicas, en las cuales se aprecian los síntomas correspondientes al órgano invadido: cistitis, obstrucciones intestinales totales o parciales, hidronefrosis, etc. Pero en líneas generales se admite que no hay proporcionalidad extre la intensidad de ios sintomas y la propagación de la enfermedad.

Indudablemente la causa más frecuente de consulta es la aparición, siempre secundaria, de dolores o sensaciones de molestia pélvica general, ya sean constantes, intermitentes, $D$ con mayor frecuencia coincidiendo o precediendo a la menstruacion, y que por regia general siempre se agrava durante ella, refiliéndose regularmante al recto e intensificada por la defecación: estas características y la tendencia a ser progresivas en su intensidad, son comunes a la mayoría de los casos.

Tales trastornos pueden ir acompañados o no de hemorragias uterinas anormales, generalmente del tipo hipermerorrea, sin que se deban producir modificaciones marcadas sobre el cicio habitual de la paciente, como lo sosttienen Calatroni y Ruiz. Los datos anteriores, unidos al retardo en el logro de concepciones, o a la clara presencia de esterilidad, constituyen la trilogía básica de la enfermedad.

Es una entidad que se presenta en la plena madurez sexual, entre la tercera y cuarta década de la vida, aunque pueden presentarse casos en las edades extromas, comprobándose aun en la menopausia, aunque muy de vez en cuando. El factor edad tiene mucha importancia, especialmente desde el punto de vista pronóstico y tratamiento a escoger. Al respecto, es muy sugestiva la estadistica de Counseller, quien recogiendo los casos encontrados y tratados en la Clínica Mayo durante los últimos diez años, concluye que, en ei $75 \%$ de los tratamientos médicos irıstituidos con un relativo éxito, se trataba de pacientes menores de treinta años. Por supuesto, estos casos sólo representaban la minima parte de los hallados, los cuales eran quirúrgicos en su gran mayoria. 
Son muy sugestivos los factores eccnómico y sccial, referidos a la incidencia de la endometriosis, ya que el mayor porcentaje de los casos se presenta en la clienteia de consultorio, siendo relativamente raros en la clientela hospitalaria. En tal concepto coinciden varios autores, algunos de los cuales han querido atribuir el fenómeno al mayor retardo matrimonial y uso más frecuente de medios anticoncepcionales en ios pacientes particulares. Estos argumentos no serían válidos en nuestro medio, donde tales factores no tendrian tanto valor. Es curioso que la frecuencia con la cual se repcrtan casos de endometriosis es, entre nosotros, muy inferior a la incidencia encontrada por autores de otros paises.

El factor racial parece influir también sobre la incidencia de la enfermedad, siendo de muy rara aparición en ia raza negra, tanto que algunos reportes, como el de Meredino y Brinik en el Harlem Hospital, encuentran sobre 4.477 intervenciones péivicas la cifra, asombrosamente baja, de $0.11 \%$ de endometriosis, contrastando con las estadísticas de la gran mayoría de los autores, cntre ellos Meigs, Counseller, Cavanagh, Tyrone, etc., quienes encontraron cifras oscilando entre el $8 \%$ y el $28 \%$.

Es de gran importancia constatar la coincidencia entre la endometriosis y las intervenciones quirúrgicas abdominaies, o las simples maniobras de dilatación y curetaje, a que previamente han sido sometidas tales pacientes. Las estadisticas varian, pero se encuentran algunas tan alarmantes como las de Mayo Clinic, que alcanzan al $46.3 \%$. De todas maneras, la gran frecuencia de la cirugia previa, soporta la opinión de que existe una verdadera endometriosis quirúrgica, hecha a manera de injerto sobre la mesa de operaciones. Por lo tanto, son obvias las recomendaciones de suaves maniobras operatorias, especialmente en pacientes jóvenes, y muy particularmente si encontramos dismenorréa de cualquier modalidad dentro de su sintomatologia previa. Son tan importantes los traumatismos de cualquier tipo, que no es nada raro encontrar entre los antecedentes persuflaciones, histerosalpingografías, y aun el uso de pesarios.

Sin duda uno de los puntos más interesantes de estudio, y que por lo tanto ha merecido multitud de trabajos, es aquel de la coexistencia de la endometriosis ccn esterilidad, infevtijidad y embarazo. Pero de la inmensa literatura al respecto, sóı se destacan algunos hechos: en primer lugar, la alta incidencia de endometriosis con esterilidad o infertilidad, no sólo en aquelios casos perfectamente explicables; en los cuales las secuelas adherenciales han causado obstrucciones tubarias, o impiden, poi fijación. la 
normal rotación ovárica en el momento de la ovulación, sino en el gran número de ocasiones con perfecta permeabizidad tubaria, estudio normal del funcionalismo ovárico, sin encontrarse ninguna causa de esterilidad, masculina o femenina, sino únicamente la presencia de una endometriosis diagnosticada clinica o quirúrgicacamente.

En segundo lugar también es indudable la existencia de endometriosis coincidiendo con embarazo, contingencia que plantea las influencias mutuas entre las dos condiciones.

Ya desde 1922 Sampson suponía que las lesiones endometriósicas serían faborablemente influenciadas por el embarazo, y posteriormente Beecham dice: "la naturaelza ha empleado un gran medio profiláctico contra la endometriosis: el embarazo". Por otra parte, Gayn y sus colaboradores encontraron que, en el 80\% de sus pacientes con endometriosis y embarazo, las molestias subjetivas desaparecían o se atenuaban notablemente y que en nirgún caso, pasado el parto, las pacientes empeoraban subjetiva u objetivamente.

Respecto a la influencia que la lesión en sí suf:e con el embarazo, hay diferencia de opiniones. Novak y De Lima, como arriba dijimos, sostienen que las caracteristicas de inmadurez del endometrio ectópico serían poco favorables a los cambios deciduales, mientras que Everett no admite lo anterior, susteniendo clinicamente la lógica de los cambios deciduales y cornprobándolos histologicamente.

El diagnóstico, muy dificultoso o imposible en algunas ocasiones, será tanto más sencillo cuanto mayor número de sintomas sean hallados, maximum si a la exploración encontramos el nódulo del tabique rectovaginal, casi patognomónico, indưración en el fondo de saco de Douglás sobre los ligamentos útero-sacros o sobre la superficie corporal posterior del útero, esvecialmente si éste es adherente o está en retroposición, o si se enciaentran quistes parauterinos u ováricos adherentes.

El diagnóstico roentgenológico se hace con mayor probabilidad en los casos de adenomiosis: la penetración del endometrio en el músculo forma estructuras tubulares en las cuales penetra el medio opaco, produciendo figuras caracteristicas, cortas, como espiculadas, extendiéndose principalmente sobre la superficie superior del úteru, variando de tamaño entre $1 \mathrm{~mm}$. y $4 \mathrm{~mm}$. y terminando por dilataciones seculares muy pequeñas; la mayor fluidez del medio de contraste (Skiodan, Medopake) proporciona figuras más nitidas. 


\section{Conducta}

En general, los tratamientos dependerán de la edad, topografía, extensión, sintomatología, etc. Como regla, en pacientes jóvenes, por intensos que sean los síntomas subjetivos, debe intentarse siempre tratamiento médico; ya sea por roentgenoterapia $u$ hormonoterapia; aquél, que gozó de un extraordinario auge, se usa en el presente cada vez menos, ya que las dosis subcastración son poco beneficiosas, $y$ frecuentemente perjudiciales, mientras que la castración por rayos $\mathrm{X}$ no presenta comparación con la quirúrgica.

Para el tratamiento hormonal son usadas las sustancias andrógenas y las estrógenas. De éstos se emplean los preparados sintéticos a dosis altas, pues se ha comprobado que las pequeñas dosis generalmente aumentan la sintomatología subjetiva; la terapéutica se hace intensiva y prolongada, hasta lograr periodos de amenorrea de tres a seis meses, por inhibición indirecta de la función ovárica a través del freno a la antero-hipófisis. La gran mayoria de los autores usan este tratamiento sólo para control temporal de la enfermedad, regnesando los sintomas al suspender la droga y no comprobándose, por lo regular, regresión alguna de las lesiones.

Entre los andrógenos se usan principalmente aquellos con menor poder virilizante, recomendándose no llegar, en ningún caso, a dosis de 350 a 400 miligramos. Frecuentemente proporcionan un gran alivio de los síntomas, en caso de lesiones poco extensas; sin embargo, no se muestran como tratamiento de escogencia, presentando, en cambio, con mucha regularidad, sintomatología desagradable.

El tratamiento quirúrgico es indicado en gran proporeión de casos, siendo también el que ocasiona un mayor nümero de resultados favorables. Siempre tratará de ser lo máiz conservador posible, muy especialmente en pacientes jóvenes con deseos de embarazo. Deben extirparse todos, o el mayor número posible de implantes, las porciones ováricas lesionadas, tratando de respetar siempre parte de uno o de los dos ovarios, completindo con una suspensión uterina y una neurectomía presacra.

Si a pesar de los procedimientos anteriores se temiere una recurnencia, debe practicarse además una neurectomía ovárica bilateral. En los casos muy avanzados, cuando no hay esperanzas 
en la cirugia conservadora, la castración seguida cie histerectomía total, es el procedimiento aconsejado.

Fara finalizar nos permitimos transcribir las conclusiones expuestas por Gardner al V Congreso Americano de Ginecologia y Obstetricia, celebrado en los finales del año pasado. Dicen:

1. La endometriosis pélvica sólo es sobrepasada por los niomas uterinos y las heridas obstétricas, como indicación para una cirugía ginecológica mayor.

2. La endometriosis, en varios grados, es encontrada en un $30 \%$ de nuestras laparotomías.

Se asocia frecuentemente con infertilidad y es, a menudo, responsable de la dismenorrea adquirida durante la vida adulta.

4. Interesa al internista, ocasionalmente, a causa" de fiebres inexplicables y más, usualmente por cuadros de colıtis espasmódicas.

5. Presenta interés para el urólogo, ya sea por cbstrucciones ureterales o por fenćmenos vesicales directos.

6. Interesa al cirujano general por los cuadros de obstrucción intestinal, por falsos ataques de apendicitis aguda y por serios problemas de diagnóstico y conducta, en ia mesa de operaciones.

7. Por lo tanto, un proceso patógeno que se ve con tanta frecuencia, que causa a las mujeres tantos trastornos y que, en ocasiones, requiere para el diagnóstico y tratamiento consultas de tantos especiailstas, es un problema fascinante para todo médico.

Observamos muy claramente, al comparar la incidencia de la enfermedad en nuestro medio, con ia traída por los autores de otros paises, en especial los americanos, una gran diferencia en las cifras globales, estando las nuestras muy por ciebajo de las otras reportadas.

Esto lo comprobamos al estudiar el excelente trabajo del profesor Arturo Aparicio (tesis de agregaturia), y los trabajos presentados al primer congreso colombiano de Ginecolcgía y Obstetricia, celebrado el año pasado en Bogotá, por los doctores Pedro Nel Cardona, de Medellín, y Hernando Amaya León, de Bogotá. Támbién en nuestro trabajo la incidencia sólo llesó al $4.4 \%$ de todo el personal hospitalizado en el servicio ginecoligico del Hospital de San Juan de Dios, Cali.

El por qué de la menos frecuencia de la endometriosis en inuestro medio podría ser atribuída, tanto a la mayor precocidad para contraer matrimonio, como al menor uso de mecidas contraceptivas, influido muy particularmente por el problema religioso, 


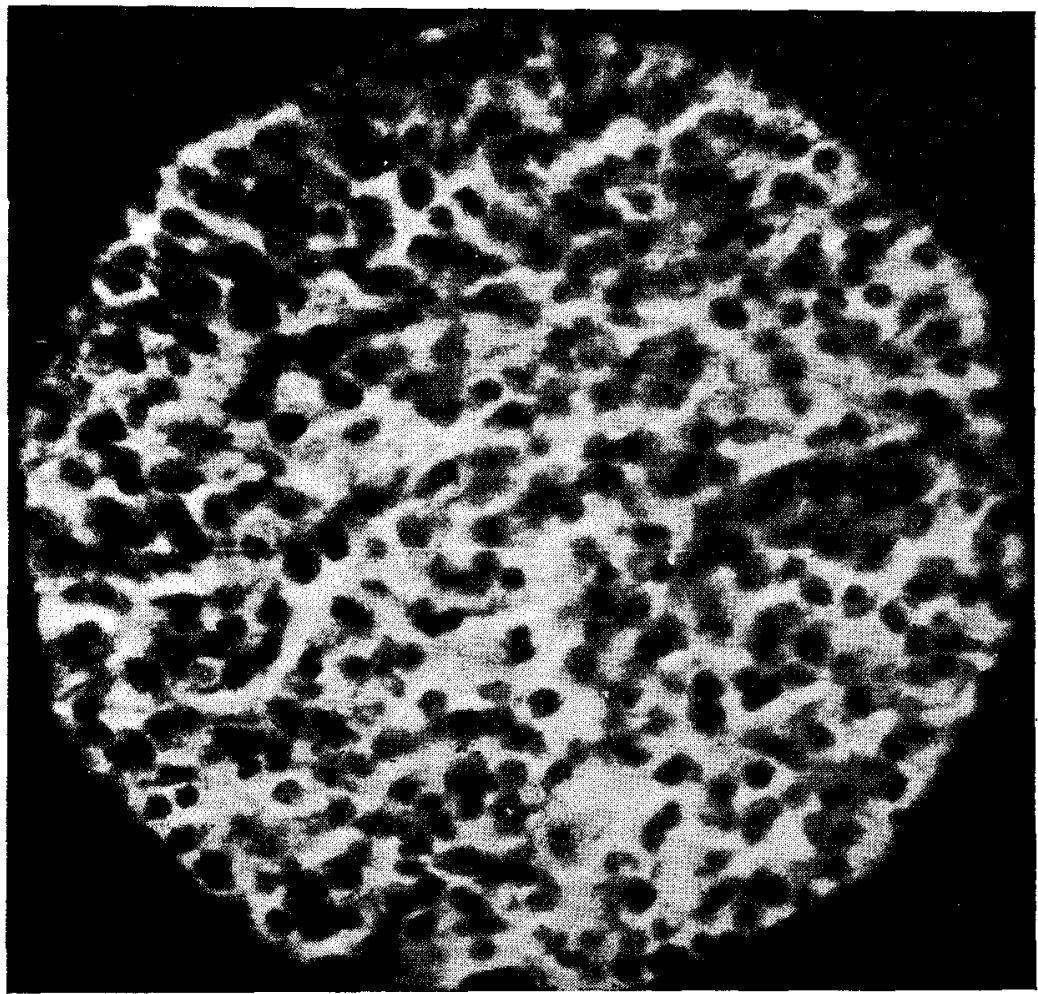

sin descartar, en forma hipotética, algún factor racial imponderable, similar al que ocasiona la mínima incidencia de estas lesiones en la raza negra. Es posible que con ei mayor interés en el tema, los diagnósticos sean más frecuentes, especialmente con la tendencia, todos los dias mayor, de controlar histopatológicamente todas las piezas extirpadas.

\section{Historias}

En el curso de dos años encontramos en el Hospital de San Juan de Dios, Cali, Servicio de Ginecologia, sobre un total de $\mathbf{5 5 0}$ casos quirúrgicos, 20 de endometriosis, o sea un $4.4 \%$, algunos diagnosticados en la mesa de operaciones y otros soin al examen anatomopatológico. 


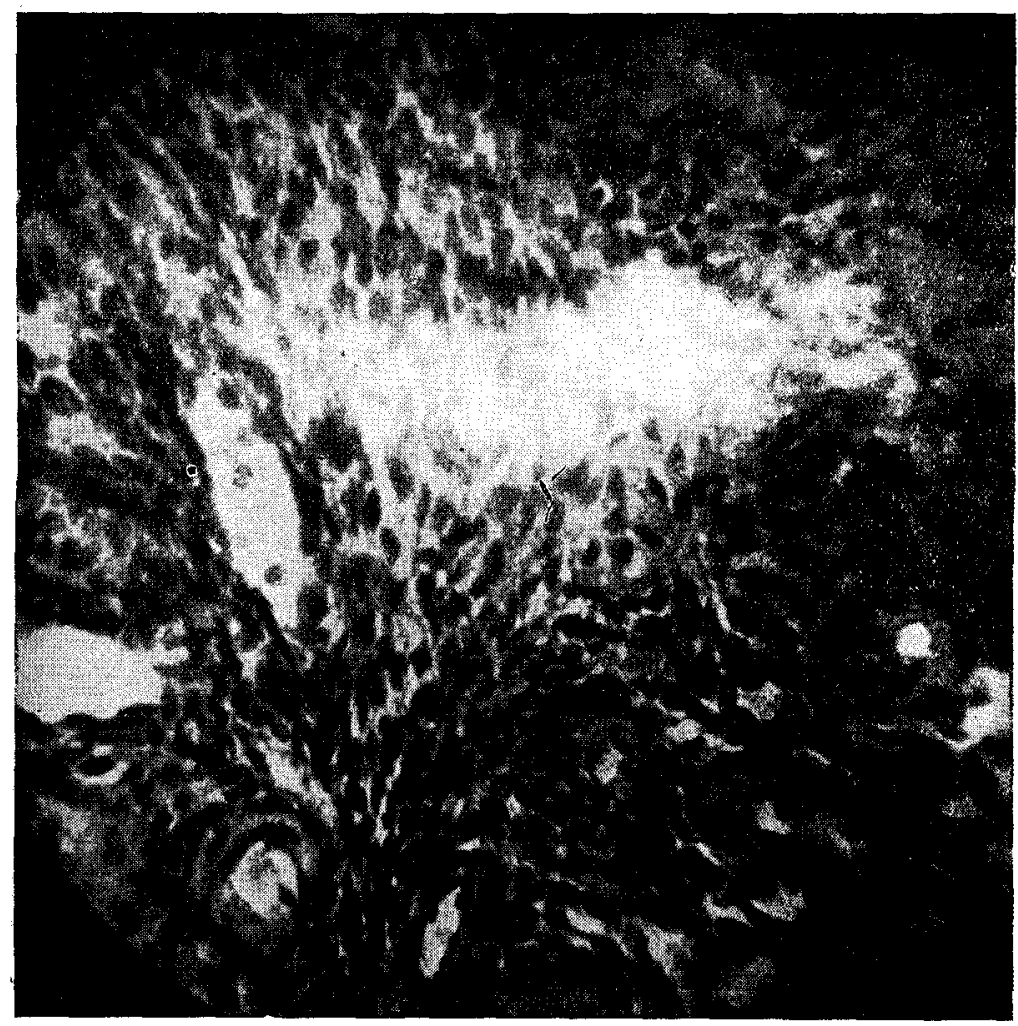

Faré un resumen rápido de tres historias clínicas de pacientes intervenidas por mí, de los cuaies poseo las respectivas placas.

En ellas sólo se observará la constancia de la dismenorrea, siempre secundaria. Los demás datos, en número tan infimo de casos, no permiten extraer ninguna conclusión.

Historia número 2072. T. V, treinta y cinco años.

Consulta por metrorragias y ligera menalgia de tipo expulsivo, presentadas por primera vez en su vida desde hace cuatro meses.

Ha padecido de enférmedades de la infancia, de tifoidea y paIudismo. Un parto hace 17 años. Menarca a los 13 años. Ciclos, 28/3-4. Operada en tres ocasiones, una de ellas dice que por quiste del ovario, pero no hay historias. 


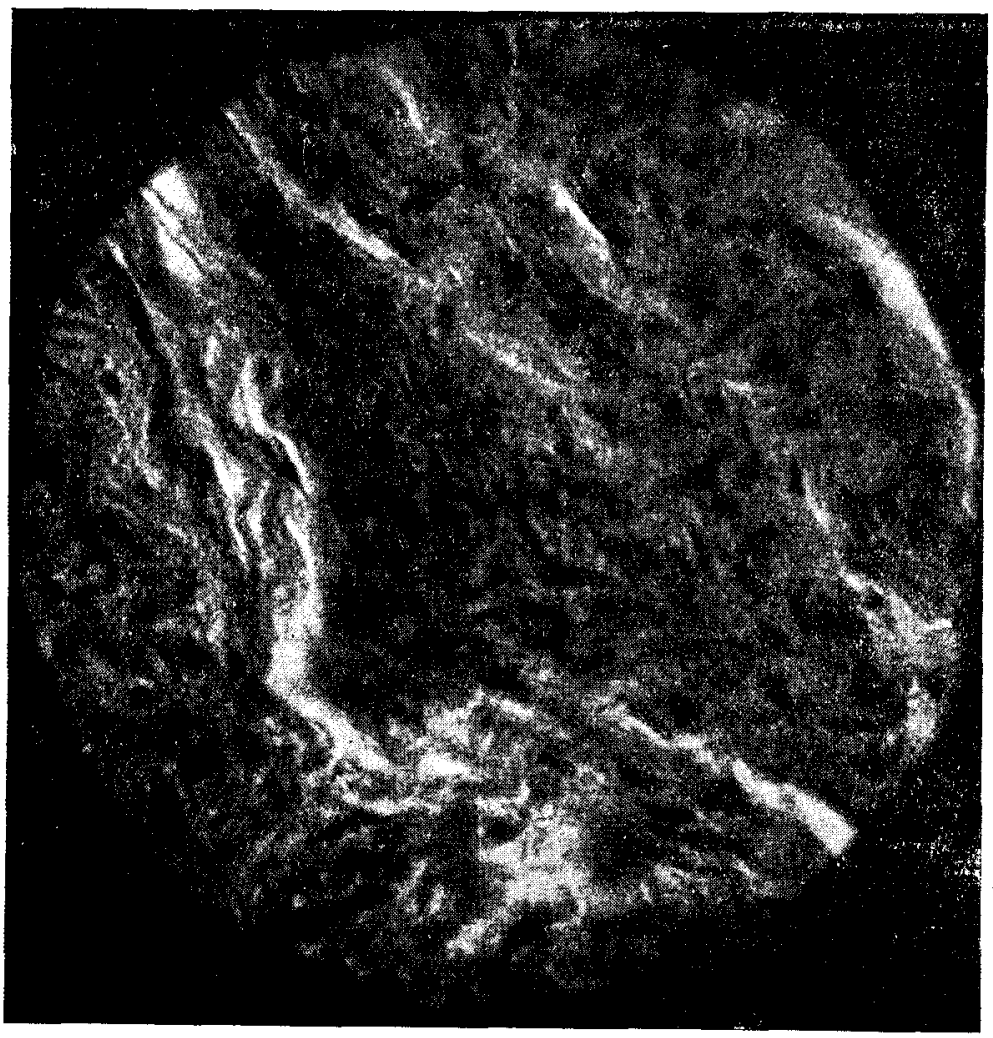

Al examen se aprecia buen estado general. En ei ginecológico sólo presenta una ligera cervicitis y una masa pelviana que parece hacer cuerpo con la matriz, dura, llegando hasta el ombligo. Se interviene con un diagnóstico de fibromatosis.

En la intervención se encuentra: pŗofundas adherencias de la vejiga y del sigmoide a la matriz, la cual se aprecia de tamaño y aspecto normal. La paciente habia sido apendicectomizada y salpingo-oforectomizada del lado derecho. Hay un gran quiste chocolate, profundamente adherido al fondo uterino y otros sobre el cuerno derecho y ovario izquierdo; éste profundamente adherido, no lográndose independizar del tejido sano, por lo cual se practica una salpingo-oforectomía izquierda, exéresis de los focos diseminados e histeroctomía total. El postoperatorio es normal y los controles normales, con medicación estrogénica de suplencia.

La patología reveló focos endométricos del Douglás, ligamento ancho y ovario, al cual corresponde la placa númers 1.993. 


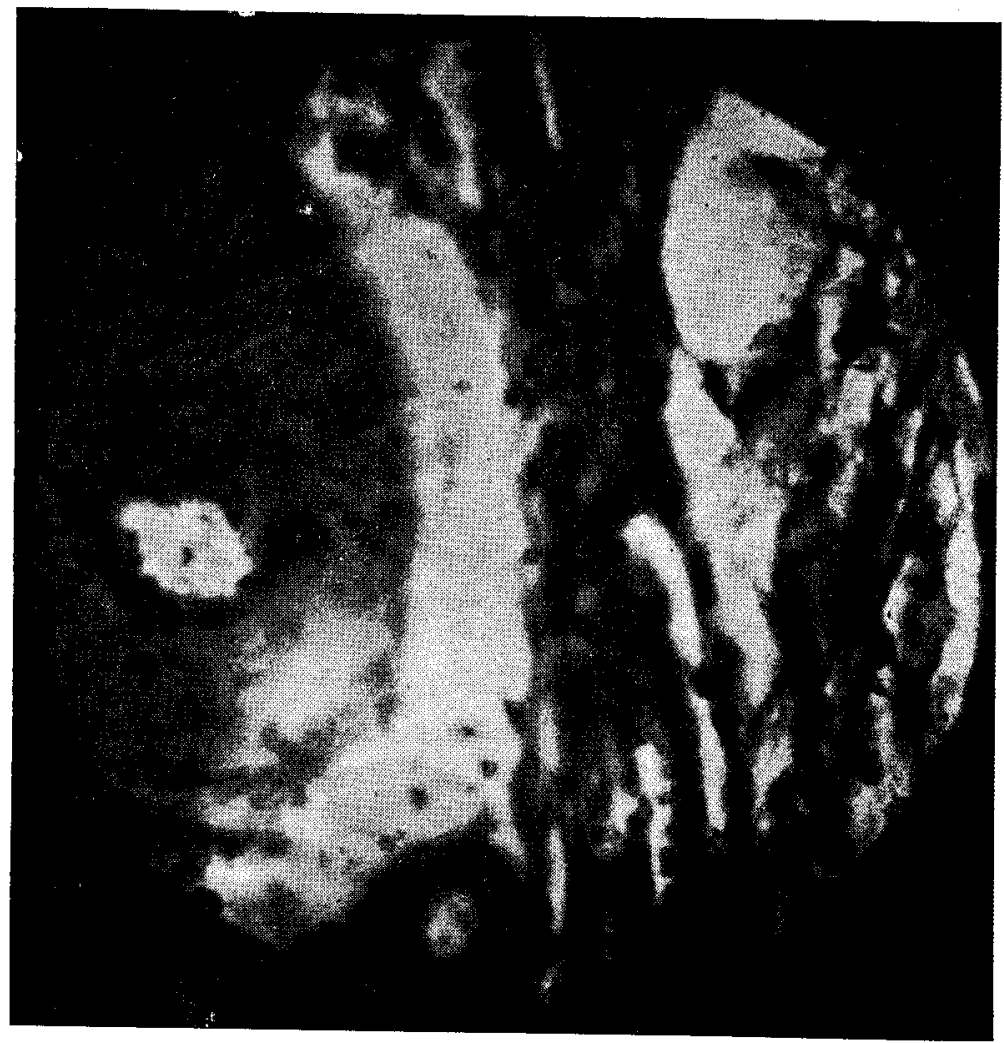

Historia número 4.001. E. S., cuarenta años. Octubre de 1952. Consulta por menometrorragias y dolores a la defecación desde hace cinco años, acompañados de aigomenorrea desde dos años.

Paciente de raza negra. Ha padecido de enfermedades de la infancia y de paludismo. Seis partos, el último hace ocho años. Menarca a los 15 años. Ciclos de 28-30 X 3-4, hasta la iniciación de la dolencia, siendo $12-30 \times 6-10$.

El examen ginecológico sólo reveló: matriz aumentada de tamaño, nodulosa, dura, poco móvil. Se opera con un diagnóstico de fibromatosis uterina.

A la intervención sólo se encuentra una matriz fibromatosa, con múltiples nódulos, intramurales y subserosos.

La patología reportó adenomiosis, a la cual corresponde la placa número 1.830. Además, fibromatosis. 


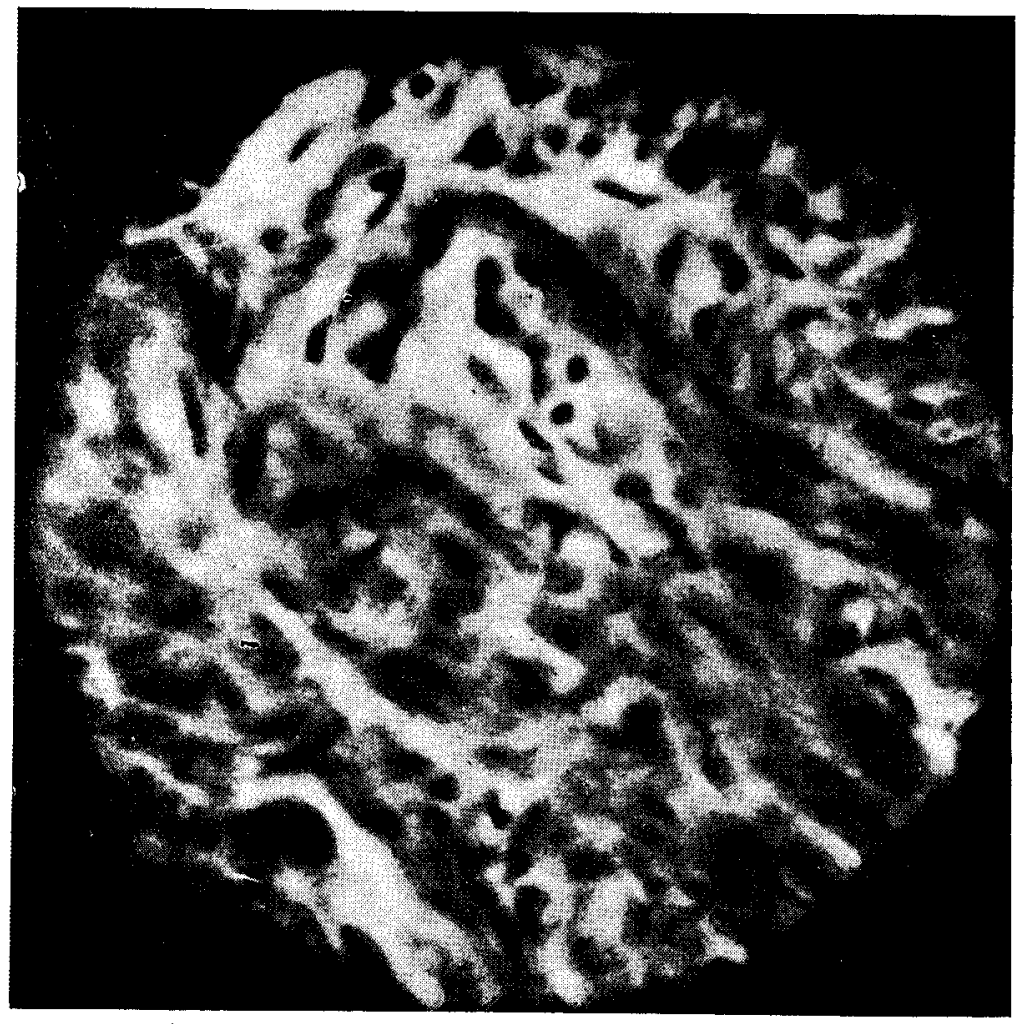

Historia número 10.076. M. J. C., diecinueve años. Septiembre de 1953 .

Consulta por dolores permanentes, que se exacerban durante los periodos menstruales, desde hace tres meses y iocalizados en la fosa iliaca izquierda; durante la semana premenstrual hay fiebre baja y flujo puruiento.

Enfermedades de la infancia. Nulípara. Menarca a los 12 años. Ciclos de 26-30 X 4-5.

Al examen sólo se aprecian los fondos laterales llenos y sensibles, especialmente el derecho; matriz de nulipara en retroversoflexión fija de primer građo. Se opera con el diagnóstico de anexitis.

A la intervención se encuentra piosalpinx derecho y adherencias ováricas bilaterales. Se practica apendicectomía, salpingecto- 
mía y hemiresección ovárica derecha, salpingolisis, liberación del ovario izquierdo, histeropexia y neurectomia presacra.

La patología reveló implantes endometrićsicos del ovario. Placa número 1.747 .

En todos ios casos no habia datos generales, o de laboratorio, que merezcan mención especial.

\section{BIBLIOGRAFIA}

E. Novak.-Textbook of Gynecology. Williams and Wikins. Second edition. pañola.

Crossen. Crossen.--Enfermedades de la mujer. Uteha. Seginda edición es-

Calatroni. Ruiz.-Terapéutica Ginecológica. El Ateneo. Cuarta edición.

Te Linde.-Operative Gynecology. Lippincot. Second' edition. .

Max Wallet.-L'hyperfolliculinie. Masson. 1946.

Raoul Palmer--La sterilité involuntaire. Masson. 1950.

J. V. Meigs.-Endometriosis, its significance. Ann. Surg. 114: 866. 1941.

A. Decker.-Culdoscopy. W. B. Saunders. 1952.

H. Klunner. W. Bartelemez.-Endometriosis in a Rheuses Monkey. Sur. gery, Obstetrics and Gynecology. 92: 6. 1951.

\section{Del American Journal of Obstetries and Gynecology}

S. L. Siegler.-Endometriosis, clinical. 61: 1. 1951.

W. V. Camanach.-Fertility in the etiology of endometric is. 61: 3. 1951.

J. W. Huffman.-Externa! endometriosis. 62: 6. 1951.

R. J. Leffler-Endometriosis with decidual raecticn in cpisiotomy scar. 62: 5. 1951.

E. S. Cohen.-Endometriosis in the omentum, suggesting cisin in ceiomic mesoderm. 62: 6. 1951.

V. S. Conseller.-A clinical and surgical review of endometriosis. 62: 4. 1951.

R. W. Kirstner.-Endometriosis occurring in a vesico-vasiral fistula. 63: 2. 1952 .

H. L. Gainey. Keeler-Endometriosis in pregnancy. Clin cal observations. 63: 3. 1952.

I. Forman--Fever in endometriosis. 63: 3, 1953.

Ch. E. Sutton. J. Hardy.-Endometricsis of the evrmiform appendix. 63: 5. 1952.

T. A. Sinclair.-The accurancy of the diagnosis of endonctriosis, 63: 6 . 1952.

B. Banney.-Endometriosis of the cervix uteri. 64: 6. 1952.

L. Zweibwl.-Primary endometriosis of the cervix. 65: 6. 1953.

E. T. Bennet.-Endcmetriosis in the older age group. 5: 1.1953.

J. W. Latchen.-Endcmetriosis of the umbilicus. 66: 1. 1953.

L. A. Gray.-Stibestrol treatment of endometriosis. 66: 2. 1953.

F. H. Falls.-Secundary dismenorreae. 66: 4. 1953. 


\section{Del Year Book of Obstetrics and Gynecology}

J. H. Blain.-Adenomiosis of the uteri. 1943.

J. C. Hirrt.-Propionato de testosterona en la endometriosis. 1943.

W. H. Brown.-Estudio de 117 casos de lesiones endometriales, rectales y sigmoideas. 1943.

S. E. Norwood.-Pregnancy after conservative surgery of endometriosis. 1953.

Scott. Te Linde.-Experimental endometriosis. 1951.

R. E. Fallas.-Endometriosis. 150 private cases. 1953.

H. C. Stearnas.-Pelvic endometriosis.

F. Brom. Fruhling.-Urologic aspects of endometriosis. 1952.

Tractions of the Fifth American Congress on Obstetrics and Gynecology. Ed. Mosby. 1953.

\section{Del Journal of Obstetries and Gin. of the Britisth Empire}

H. H. Ware-Endometriosis and pregnancy. Cit. LVIII: 1. 1951.

P. L. Krohn,-Endometriosis and supernemmerary ectoplc cvarian tissue. LVIII: 3.1951.

C. T. Javert.-Observations of the patology and spread based of theory of bening metastasis. LIX: 3. 1952.

M. P. Emprey.-Estromatous endometriosis. LIX: 6. 1952.

R. H. Hays.--Endometriosis, its management and end results. Cit. LIX: 4. 1952 .

L. M. Hurxthal.-Treatmen of endometriosis with large doses of strogens. Cit. LX: 2. 1953.

H. Frederikson.-Conservative surgery in cvarian endometriosis. Cit. IrX: 2. 1953 .

A. Alvarez Bravo.-Endometriosis. Gimecología y Obsteuricia de México. IV: 2_3. 1949 .

Arrighi. Baratelli.--Endometriosis vesical. Obstetricia y Ginecología latinoamericanas. IX: 3. 1951. 